\title{
Acute heart failure admissions in New South Wales and the Australian Capital Territory: the NSW HF Snapshot Study
}

Phillip J Newton

RN, PhD'

Patricia M Davidson $\mathrm{RN}, \mathrm{PhD}^{1,2}$

Christopher M Reid

$\mathrm{PhD}^{3,4}$

Henry Krum*

MB BS, PhD, FRACP

Christopher Hayward

David W Sibbritt

Emily Banks MBBS, PhD, FAFPHM

Peter S MacDonald MBBS, FRACP, PhD

On behalf of the NSW Heart Failure Snapshot Investigators (full list in the online Appendix)

1 Centre for Cardiovascular and Chronic Care, University

of Technology Sydney. Sydney, NSW.

2 Johns Hopkins University, Baltimore, Md, USA

3 Curtin University. Perth, WA

4 Monash Centre of Cardiovascular Research and Education in Therapeutics, Monash University, Melbourne, VIC

5 St Vincent's Hospital, Sydney, NSW.

6 Australian Research Centre in Complementary and Integrative Medicine, University of Technology Sydney, Sydney, NSW.

7 National Centre for Epidemiology and Population Health Australian National University,
Unstralian National Canberra, ACT

* Deceased.

phillip.newton@ uts.edu.au

doi: 10.5694/mjal5.00801
$\mathrm{H}$ eart failure (HF) is a major burden on the health care system, and much of this burden is associated with the high rates of admission to hospital for managing episodes of acute decompensation. Based on the expected rise in the number of patients with $\mathrm{HF}$ as the population ages, it is likely that the use of hospital resources will continue to increase. ${ }^{1}$ Detailed data on hospital admissions for HF are therefore of considerable interest, including measures of morbidity, case load and costs related to HF.

Despite the importance of national data for quantifying HF and understanding its management and outcomes, such data are not currently available. Lack of funding, difficulties with case definition, and cross-jurisdictional problems have contributed to this failure. ${ }^{2}$ To facilitate a national approach to understanding $\mathrm{HF}$ and improving outcomes, the New South Wales HF Snapshot therefore sought to provide detailed representative data on hospital admissions for acute HF.

\section{Methods}

The NSW HF Snapshot was a prospective audit of consecutive patients who presented with acute HF and were admitted to 24 public hospitals (13 metropolitan and 11 rural) in NSW and the Australian Capital Territory during a onemonth period. Each hospital had an attending cardiologist who was responsible for confirming the admission diagnosis. The snapshot methodology was based on a similar audit of patients with acute coronary syndrome. ${ }^{3}$

Data were collected from patient interviews, case note reviews and electronic database review. A twopage paper-based case report form with a standardised completion

Abstract

Objective: The primary aim of the NSW Heart Failure (HF) Snapshot was to obtain a representative cross-sectional view of patients with acute HF and their management in New South Wales and Australian Capital Territory hospitals.

Design and setting: A prospective audit of consecutive patients admitted to 24 participating hospitals in NSW and the ACT with a diagnosis of acute HF was conducted from 8 July 2013 to 8 August 2013.

Results: A total of 811 participants were recruited (mean age, $77 \pm 13$ years; $58 \%$ were men; $42 \%$ had a left ventricular ejection fraction $\geq 50 \%$ ). The median Charlson Comorbidity Index score was 3, with ischaemic heart disease (56\%), renal disease (55\%), diabetes (38\%) and chronic lung disease (32\%) the most frequent comorbidities; $71 \%$ of patients were assessed as frail. Intercurrent infection (22\%), non-adherence to prescribed medication $(5 \%)$ or to dietary or fluid restrictions (16\%), and atrial fibrillation/flutter (15\%) were the most commonly identified precipitants of HF. Initial treatment included intravenous diuretics (81\%), oxygen therapy (87\%), and bimodal positive airways pressure or continuous positive airways pressure ventilation (17\%). During the index admission, $6 \%$ of patients died. The median length of stay in hospital was 6 days, but ranged between 3 and 12 days at different hospitals. Just over half the patients (59\%) were referred to a multidisciplinary HF service. Discharge medications included angiotensin-converting enzyme inhibitors/angiotensin receptor blockers (59\%), $\beta$-blockers (66\%) and loop diuretics (88\%).

Conclusions: Patients admitted to hospital with acute HF in NSW and the ACT were generally elderly and frail, with multiple comorbidities.

Evidence-based therapies were underused, and there was substantial interhospital variation in the length of stay. We anticipate that the results of the HF Snapshot will inform the development of strategies for improving the uptake of evidence-based therapies, and hence outcomes, for HF patients.

note captured key demographic and clinical data for each patient. To standardise case and data definitions, two face-to-face meetings with representatives from all participating hospitals were held before conducting the snapshot study.

The snapshot investigation was approved by the St Vincent's Hospital Human Research Ethics Committee (reference number, LNR/13/ SVH/84). All 24 hospitals who participated had received institutional governance approval. An optout consent approach was adopted to maximise the likelihood of capturing all patients admitted to the participating hospitals. The ethics approval for the investigation did not include the capture of data from non-participating hospitals, so the admission and discharge data for patients who had been transferred from or discharged to a nonparticipating hospital were based on the first and last available results at the participating hospital.

\section{Patient eligibility}

Patients were eligible to participate in the study if they were admitted to a participating hospital between 9 am on 8 July 2013 and 9 am on 8 August 2013, and had presented with first onset HF or acute decompensation of chronic HF. The admission diagnosis was based on clinical symptoms, signs and investigations consistent 
with acute $\mathrm{HF}$, as determined by the site investigator. ${ }^{4}$

\section{Measures}

Patient data collected included demographic characteristics, medical history, clinical findings at presentation, clinical management (including pathology and cardiac investigations), acute treatment, admission and discharge medications, length of stay, and outcome of hospitalisation (including in-hospital mortality and clinical status at discharge). Classification as HF with reduced ejection fraction (HFREF) or HF with preserved ejection fraction (HFPEF) was based on the most recent assessment of left ventricular ejection fraction (LVEF), with an LVEF of at least $50 \%$ the criterion for HFPEF. Information on specific comorbidities was collected, as were data for the updated Charlson Comorbidity Index. ${ }^{5}$ Frailty was measured with the Survey of Health, Ageing and Retirement in Europe Frailty Index. ${ }^{6}$ In addition to patient-level data, all hospitals were asked to complete a survey describing their model of care, clinical service availability, and workforce characteristics. Hospitalisation and mortality outcomes were collected 30 days and 12 months after discharge by telephone follow-up and hospital electronic database review; these data will be reported in a separate article.

\section{Statistical analyses}

Dichotomous variables are reported as numbers and percentages, and were compared using either $\chi^{2}$ tests or Fisher exact tests. Continuous variables are reported as means and standard deviations or as medians and interquartile ranges. Betweengroup analyses (HFPEF $v$ HFREF) tested differences in demographic and clinical characteristics. As the evidence base for pharmacotherapy in HFREF is derived primarily from trials in patients with an LVEF $<40 \%$, we compared the rate of pharmacotherapy in patients with an LVEF $\geq 40 \%$ or $<40 \%$. Patients for whom LVEF was not reported were excluded from the between-group comparisons. Further analyses compared de novo HF and decompensated HF patients.

\section{Results}

\section{Baseline demographic factors}

In total, 811 patients were recruited. The average number recruited per site was 34 (range, 4-81; Box 1). Their mean age was $77 \pm 13$ years; most were men $(58 \%)$ and born in Australia (59\%). The majority (68\%) had experienced HF prior to their admission during the study period. Most patients presented with comorbidities, including ischaemic heart disease $(56 \%)$, renal disease $(55 \%)$ and diabetes (38\%). The median Charlson Comorbidity Index score was 3 . The most frequently attributed HF aetiologies were ischaemic heart disease (43\%) and hypertension (25\%).

Fifty-eight per cent of the patients presented with HFREF, $42 \%$ with HFPEF. Patients who presented with HFPEF were on average older, and were more likely than those with HFREF to be female and have hypertension as the antecedent cause for $\mathrm{HF}$ (each $P<0.001$ ). The Charlson Comorbidity Index scores were similar in the two groups, but the distribution of comorbidities was different; there were higher rates of pulmonary disease in the HFPEF patients $(P=0.015)$ and of ischaemic heart disease in HFREF patients $(P<0.001)$. Seventy patients $(9 \%)$ had an implantable cardioverterdefibrillator (including 61 patients with HFREF), and 133 (17\%) had permanent pacemakers (Box 2).

The most frequently identified precipitants of admission were infection (22\%) and non-adherence to medication $(5 \%)$ or to dietary or fluid restrictions $(16 \%)$. The first recorded rhythms on admission were sinus rhythm (42\%) and atrial fibrillation/ flutter $(42 \%)$. Patients with HFPEF were significantly more likely to be in atrial fibrillation than those with HFREF ( $48 \%$ v 36\%; $P=0.047)$. The mean first recorded blood pressure was $136 \pm 33 / 77 \pm 36 \mathrm{mmHg}$. Those with HFPEF had a significantly higher systolic blood pressure at admission $(P<0.001)$.

\section{Admission pathway}

Most patients (93\%) were admitted after an emergency department presentation. The breakdown according to admitting specialties is shown in Box 3. Eight per cent of patients were admitted to a dedicated HF service, $62 \%$ to a general cardiology unit, $16 \%$ to a general medical unit, and $8 \%$ to a geriatric service. Patients with HFREF were more likely to be
1 Number of patients recruited at each hospital for the NSW Heart Failure Snapshot study*

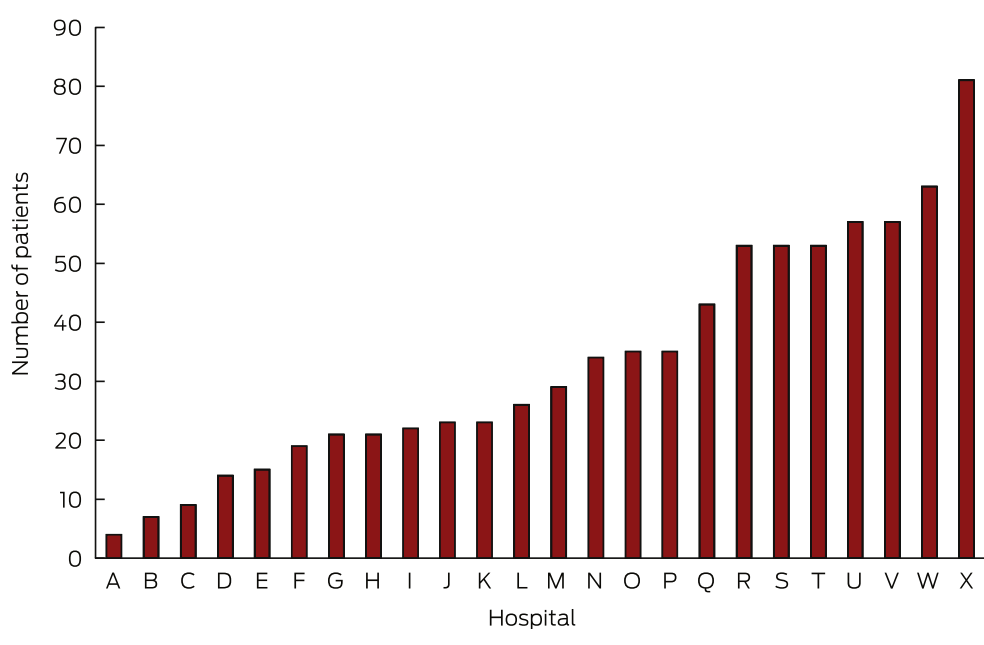

* Each site was assigned an alphabetic code to preserve anonymity, with the smallest site labelled $A$ and the largest $X$. 
2 Baseline demographic characteristics, past medical history, clinical findings at presentation and cardiac investigations for the 811 study participants*

\begin{tabular}{|c|c|c|c|c|}
\hline & Total sample & HFREF & HFPEF & $P$ \\
\hline Number of patients & 811 & 433 & 318 & \\
\hline \multicolumn{5}{|l|}{ Demographic characteristics } \\
\hline Sex (men) & $465(58 \%)$ & $295(69 \%)$ & $146(46 \%)$ & $<0.001$ \\
\hline Age, years (mean $\pm \mathrm{SD}$ ) & $77 \pm 13$ & $75 \pm 14$ & $79 \pm 12$ & $<0.001$ \\
\hline Lives alone & $272(35 \%)$ & $151(36 \%)$ & $101(33 \%)$ & 0.328 \\
\hline Nursing home resident (at time of admission) & $80(11 \%)$ & $38(10 \%)$ & $33(11 \%)$ & 0.501 \\
\hline Born in Australia & $472(59 \%)$ & $255(59 \%)$ & $182(57 \%)$ & 0.502 \\
\hline \multicolumn{5}{|l|}{ Past medical history } \\
\hline Previous chronic heart failure & $540(68 \%)$ & $306(72 \%)$ & $194(62 \%)$ & 0.008 \\
\hline Ischaemic heart disease & $445(56 \%)$ & $265(62 \%)$ & $152(49 \%)$ & $<0.001$ \\
\hline Renal disease & $433(55 \%)$ & $256(54 \%)$ & $177(57 \%)$ & 0.601 \\
\hline Diabetes & $304(38 \%)$ & $165(39 \%)$ & $120(39 \%)$ & 0.211 \\
\hline Pulmonary disease & $251(32 \%)$ & $117(29 \%)$ & $113(37 \%)$ & 0.015 \\
\hline Charlson Index score (median [IQR]; range) & $3[2-5] ; 0-16$ & $3[2-5] ; 0-16$ & $3[2-5] ; 0-14$ & 0.914 \\
\hline \multicolumn{5}{|l|}{ Heart failure aetiology } \\
\hline Hypertension & $204(25 \%)$ & $85(20 \%)$ & $104(33 \%)$ & $<0.001$ \\
\hline Ischaemic heart disease & $346(43 \%)$ & $233(54 \%)$ & $90(28 \%)$ & $<0.001$ \\
\hline Cardiomyopathy & $121(15 \%)$ & $93(22 \%)$ & $24(8 \%)$ & $<0.001$ \\
\hline Other & $236(29 \%)$ & $87(20 \%)$ & $133(42 \%)$ & $<0.001$ \\
\hline Implantable cardioverter-defibrillator & $70(9 \%)$ & $61(15 \%)$ & $6(2 \%)$ & $<0.001$ \\
\hline Permanent pacemaker & $133(17 \%)$ & $86(20 \%)$ & $40(13 \%)$ & 0.008 \\
\hline Echocardiogram $^{\dagger}$ & $701(96 \%)$ & $405(96 \%)$ & 296 (96\%) & 0.781 \\
\hline
\end{tabular}

Clinical findings at presentation

Precipitant for admission

0.047

Ischaemia

$98(13 \%)$

$71(16 \%)$

27 (9\%)

Infection

169 (22\%)

95 (21\%)

$74(25 \%)$

Rhythm abnormality

$112(15 \%)$

$63(14 \%)$

$49(16 \%)$

$41(5 \%)$

$21(5 \%)$

$20(7 \%)$

Non-adherence to dietary or fluid restrictions

$124(16 \%)$

$82(18 \%)$

$42(14 \%)$

Other

$214(28 \%)$

$127(28 \%)$

$87(29 \%)$

Rhythm on admission

Sinus rhythm

Atrial fibrillation/flutter

$330(42 \%)$

$183(44 \%)$

0.004

$330(42 \%)$

$152(36 \%)$

$124(40 \%)$

$136 \pm 33$

$132 \pm 33$

149 (48\%)

Systolic blood pressure on admission, $\mathrm{mmHg}$ $($ mean $\pm \mathrm{SD})$

Diastolic blood pressure on admission, $\mathrm{mmHg}$ (mean $\pm \mathrm{SD})$

$$
77 \pm 36
$$

$79 \pm 43$

$141 \pm 32$

$<0.001$

$\mathrm{HFREF}=$ heart failure with reduced ejection fraction; $\mathrm{HFPEF}=\mathrm{HF}$ with preserved ejection fraction; IQR = interquartile range; $\mathrm{SD}=$ standard deviation. * Left ventricular ejection fraction was not reported for 60 patients; the data for these patients were included in the total sample analysis, but not in the separate analyses of the HFREF and HFPEF groups. † Investigators were asked to record whether subjects had undergone echocardiography either during the index admission or prior to admission as part of the investigation for clinically diagnosed heart failure.

admitted to a cardiology service, whereas HFPEF patients were more likely to be admitted to a general medical unit.

\section{Treatment received during admission}

Box 4 summarises the treatments received by patients during their hospital stay. The most common therapies were oxygen therapy $(87 \%)$ and intravenous diuretics (81\%). Intravenous inotropic agents and 
3 The admitting specialties for the 811 study participants

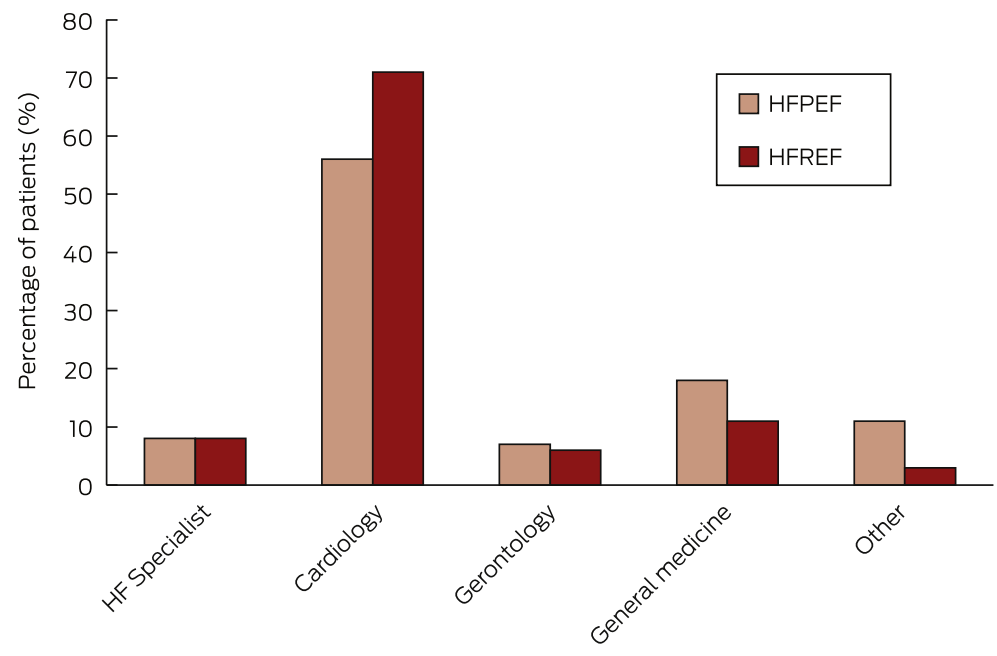

$\mathrm{HF}=$ heart failure; HFREF = heart failure with reduced ejection fraction; HFPEF = heart failure with preserved ejection fraction. $\bullet$

glyceryl trinitrate were administered to $8 \%$ and $7 \%$ of patients respectively. Non-invasive ventilation was used for $17 \%$ of patients. Apart from oxygen therapy $(89 \%$ v $83 \% ; P=0.013)$ and inotropic infusions (10\% v6\%; $P=0.032)$, there were no statistically significant differences in the treatment received by patients with HFREF and HFPEF.

\section{Pharmacotherapy}

Box 5 shows the proportion of patients receiving cardiovascular pharmacotherapy at admission and discharge for those with an LVEF $\geq 40 \%$ or $<40 \%$. With the exception of loop diuretics $(64 \%$ at admission $v 88 \%$ on discharge; $P<0.001)$, there was little or no change in medication use between admission and discharge for those with an LVEF $\geq 40 \%$. There was increased prescription of loop diuretics $(69 \%$ at admission $v 88 \%$ on discharge; $P<0.001$ ) and mineralocorticoid antagonists (26\% v 45\%; $P=0.010)$ for patients with an LVEF under $40 \%$, and also a non-significant increase in the use of $\beta$-blockers (60\% v 78\%; $P=0.065)$.

For patients presenting with de novo $\mathrm{HF}$ and an LVEF $<40 \%$, there was increased prescription of angiotensin-converting enzyme inhibitors (ACEIs)/angiotensin receptor blockers (ARBs) $(42 \%$ at admission $v 66 \%$ on discharge; $P=0.002)$. However, the prescription of ACEIs/ARBs for those with decompensated $\mathrm{HF}$ declined from $58 \%$ to $47 \%(P=0.010)$.

For patients with de novo HF, the frequency of combination therapy (ACEIs/ARBs with $\beta$-blockers: $24 \%$ to $60 \%$ ) and triple therapy (ACEIs/ARBs, $\beta$-blocker and mineralocorticoid antagonist: $4 \%$ to $19 \%$ ) increased between admission and discharge. In patients with decompensated $\mathrm{HF}$, there was no change in the frequency of either double $(43 \%$ v $45 \%)$ or triple therapy $(16 \%$ v $21 \%)$. The total daily diuretic dose

\begin{tabular}{|c|c|c|c|c|}
\hline & Total sample & HFREF & HFPEF & $P$ \\
\hline Number of patients & 811 & 433 & 318 & \\
\hline Intravenous diuretics & $643(81 \%)$ & $342(81 \%)$ & $250(80 \%)$ & 0.824 \\
\hline Intravenous glyceryl trinitrate & $53(7 \%)$ & $26(6 \%)$ & $22(7 \%)$ & 0.670 \\
\hline Oxygen therapy & $673(87 \%)$ & $369(90 \%)$ & $254(83 \%)$ & 0.008 \\
\hline Intravenous inotrope & $63(8 \%)$ & $42(10 \%)$ & $17(6 \%)$ & 0.023 \\
\hline Dialysis & $16(2 \%)$ & $9(2 \%)$ & $7(2 \%)$ & 0.964 \\
\hline $\begin{array}{l}\text { Intra-aortic balloon pump/extracorporeal } \\
\text { membrane oxygenation }\end{array}$ & $8(1 \%)$ & $7(2 \%)$ & $1(0 \%)$ & 0.081 \\
\hline $\begin{array}{l}\text { Continuous positive airways pressure/bimodal } \\
\text { positive airways pressure }\end{array}$ & $133(17 \%)$ & $73(18 \%)$ & $50(16 \%)$ & 0.573 \\
\hline Cardiopulmonary resuscitation & $21(3 \%)$ & $12(3 \%)$ & $7(2 \%)$ & 0.578 \\
\hline Mechanical ventilation & $29(4 \%)$ & $18(4 \%)$ & $8(3 \%)$ & 0.186 \\
\hline Referred for heart transplant assessment & $17(2 \%)$ & $13(3 \%)$ & $4(1 \%)$ & 0.101 \\
\hline Referred to multidisciplinary care team & $458(59 \%)$ & $261(63 \%)$ & $173(56 \%)$ & 0.055 \\
\hline
\end{tabular}



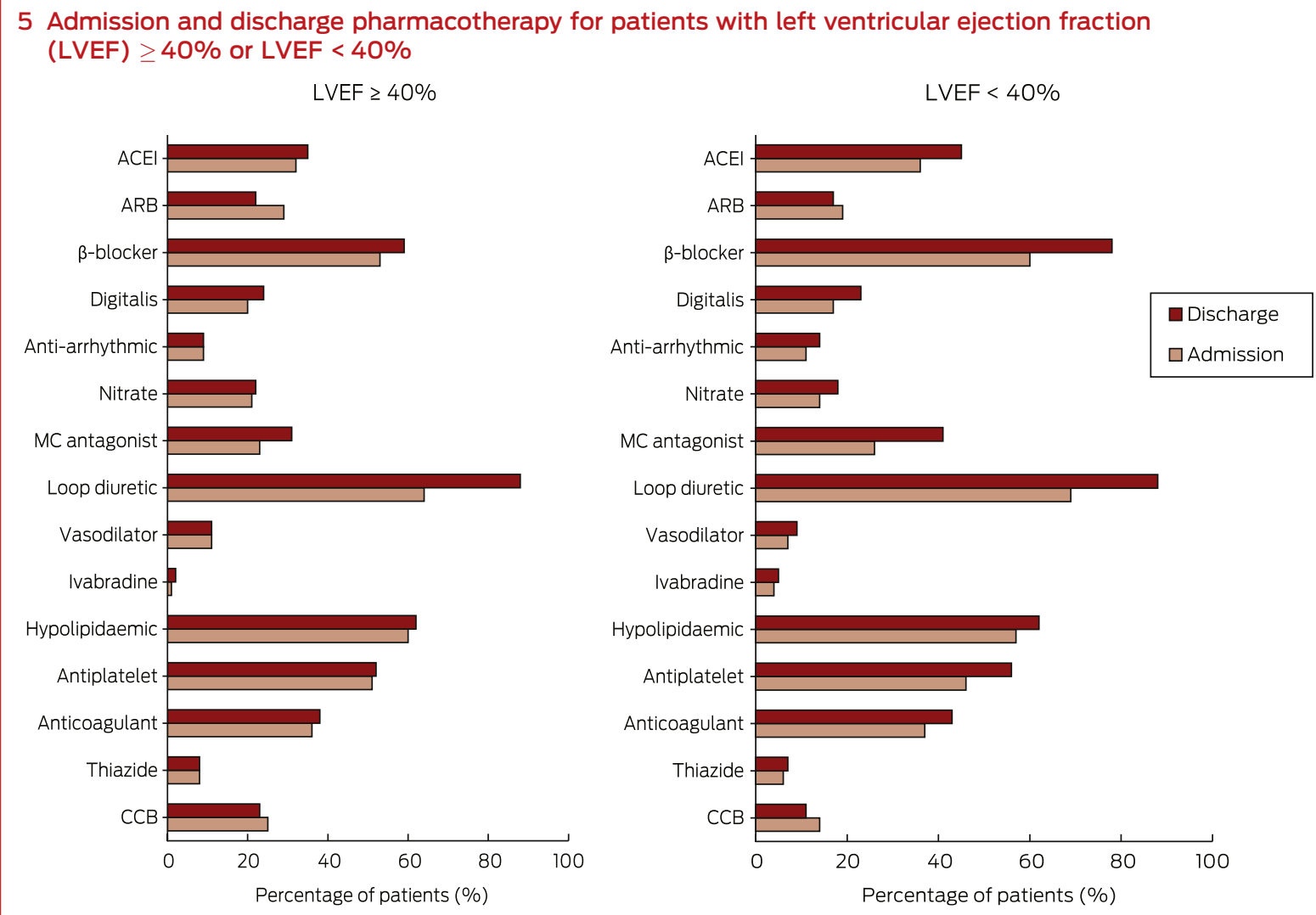

$\mathrm{ACEI}=$ angiotensin-converting enzyme inhibitor; $\mathrm{ARB}=$ angiotensin receptor blocker; $\mathrm{CCB}=$ calcium channel blocker; $\mathrm{MC}$ antagonist $=$ mineralocorticoid receptor antagonist.

(frusemide equivalent) at discharge was $75 \pm 72 \mathrm{mg}$. At discharge, patients were taking an average of $10 \pm 4$ agents a day, requiring them to swallow $13 \pm 7$ pills each day.

\section{Pre-discharge clinical status and discharge outcome}

Forty-five patients $(6 \%)$ died during the index admission. Of 766 patients who survived to discharge, 557 (73\%) underwent frailty assessment, of whom a large proportion (71\%) were assessed as being frail. On discharge, the condition of most patients $(83 \%)$ was classified as New York Heart Association class II (mild symptoms) or III (marked limitations of activity) (Box 6). The median length of stay was 6 days for patients who survived to discharge, and 7 days for those who died during admission. As shown in Box 7, the median length of stay ranged between 3 and 12 days for individual hospitals. Most patients $(74 \%)$ were discharged home. Just over half the patients
(59\%) were referred to a multidisciplinary HF service.

\section{Discussion}

The NSW HF Snapshot provides a unique window onto the characteristics of patients admitted with acute HF to public hospitals in urban and rural NSW and the ACT. The opt-out approach to patient consent enabled consecutive patients to be enrolled, limiting selection bias and providing a real world picture of patients and their care.

The mean age of patients enrolled in the NSW HF Snapshot was greater than that of patients enrolled in other international HF registries, ${ }^{7-17}$ although it was comparable with that of patients from Australian sites that participated in the Acute Decompensated Heart Failure Registry - Asia Pacific (ADHERE-AP). ${ }^{18}$ The patients were also similar in many other key demographic and clinical characteristics to those in ADHERE-AP. It is encouraging that the proportion of patients who had an echocardiogram in our study was much higher than reported in previous Australian studies. ${ }^{18,19}$

The most common precipitants of acute HF identified were infection and non-adherence to medications or to dietary or fluid restrictions, each of which accounted for about one-fifth of hospitalisations. Similar rates for these precipitants have been reported by other registries. ${ }^{7,20}$ The high rate of infection-related HF is consistent with the fact that the snapshot was conducted during mid-winter. We did not record immunisation history, but this would be a valuable addition to a future survey. The high rate of non-adherence as a precipitant is disappointing, but underscores the critical need for disease management interventions. $^{21}$ The prevalence of atrial fibrillation or flutter on admission was higher than recorded by 


\begin{tabular}{|c|c|c|c|c|}
\hline Pre-discharge assessment & Total sample & HFREF & HFPEF & $P$ \\
\hline Frailty $(n=557)$ & & & & 0.038 \\
\hline Not frail & $45(8 \%)$ & $29(9 \%)$ & $13(6 \%)$ & \\
\hline Pre-frail & $115(21 \%)$ & $76(24 \%)$ & $35(17 \%)$ & \\
\hline Frail & $397(71 \%)$ & $214(67 \%)$ & $164(77 \%)$ & \\
\hline New York Heart Association class $(n=706)^{\dagger}$ & & & & 0.103 \\
\hline Class I & $70(10 \%)$ & $26(7 \%)$ & $33(12 \%)$ & \\
\hline Class II & $368(52 \%)$ & $208(55 \%)$ & $145(52 \%)$ & \\
\hline Class III & $220(31 \%)$ & $117(31 \%)$ & $86(31 \%)$ & \\
\hline Class IV & $48(7 \%)$ & $30(8 \%)$ & $15(5 \%)$ & \\
\hline \multicolumn{5}{|l|}{ Outcome $(n=783)^{\dagger}$} \\
\hline Discharged home & $582(74 \%)$ & $352(75 \%)$ & $230(74 \%)$ & 0.271 \\
\hline Discharged to residential aged care facility & $71(9 \%)$ & $36(8 \%)$ & $35(11 \%)$ & \\
\hline Transferred to another medical facility & $85(11 \%)$ & $56(12 \%)$ & $29(9 \%)$ & \\
\hline Died & $45(6 \%)$ & $28(6 \%)$ & $17(6 \%)$ & \\
\hline
\end{tabular}

HFREF = heart failure with reduced ejection fraction; HFPEF = heart failure with preserved ejection fraction. * Left ventricular ejection fraction was not reported for 60 patients; the data for these patients were included in the total sample analysis, but not in the separate analyses of the HFREF and HFPEF groups. †Reduced numbers are the result of missing data in the case report forms for these data items. New York Heart Association class was recorded only for hospital survivors.

most acute HF registries, and may be partly explained by the older age of patients in the NSW HF Snapshot. Interestingly, although atrial fibrillation/flutter was detected in $42 \%$ of patients on admission, it was thought to have been the precipitant of HF in only $15 \%$ of patients.

In-hospital pharmacotherapy was similar to that reported by other major international acute $\mathrm{HF}$

7 Median length of hospital stay*

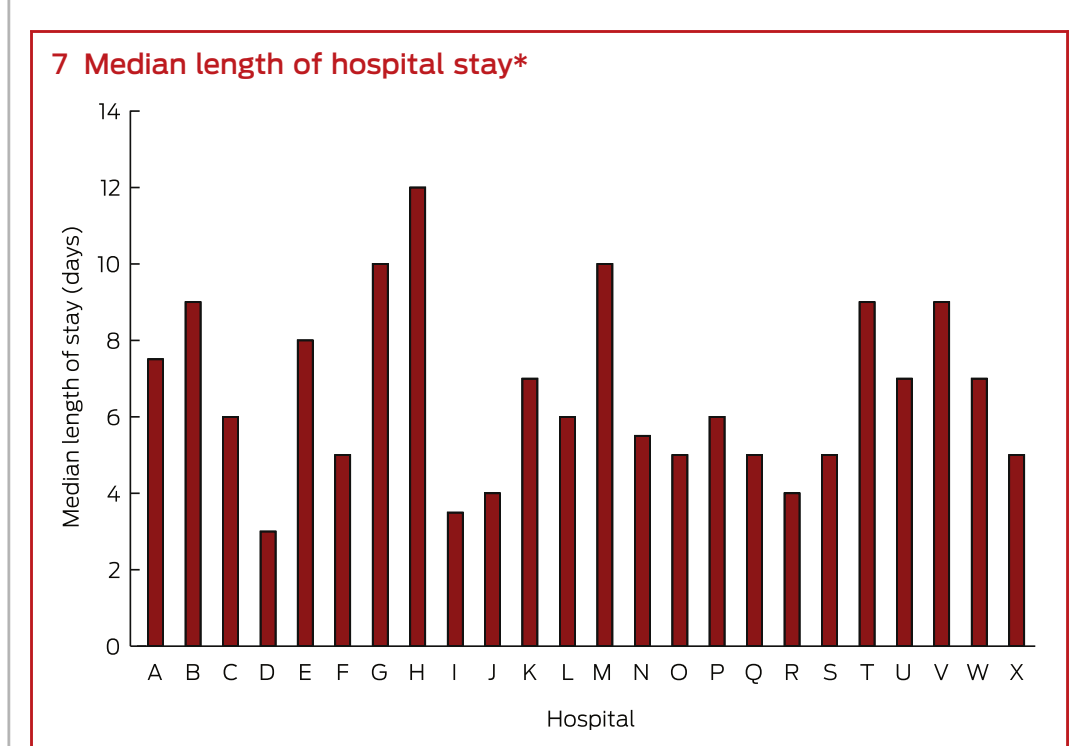

* Each site was assigned an alphabetic code to preserve anonymity, with the smallest site labelled $A$ and the largest $X$. registries, ${ }^{17,18,22}$ highlighting the lack of new therapies for acute HF. Intravenous vasodilators and inotropic agents were each administered to less than $10 \%$ of patients, consistent with recent international trends finding that use of these therapies is declining. ${ }^{23,24}$ The frequency of mechanical ventilation support (mainly non-invasive) was about $20 \%$, similar to figures reported by international HF registries. $7,9,23$ 
with frailty. ${ }^{26}$ We chose to use a simple measure of frailty, consisting of five questions and a measure of handgrip strength. ${ }^{6}$ This was, however, developed for primary care settings, so we cannot rule out the possibility that we overestimated the prevalence of frailty. Our data are among the first to report the prevalence of frailty in patients admitted to hospital with HF.

The variation in the length of stay could be explained by a number of factors. The NSW HF Snapshot was conducted across a broad range of institutions, from small rural hospitals to large tertiary referral centres. Variations in patient mix, available resources, and admitting speciality are just some of the factors that may have contributed to the variation. Future analyses will explore the impact of these and other factors on the length of stay.

Disease registries provide a valuable tool for capturing data over time, and allow for the evaluation of the quality of care and outcomes. Ideally, a registry would capture all relevant patients, but for common conditions, such as HF, this is usually not the case. A major criticism of registries is that they are expensive and labourintensive to run and maintain. The snapshot methodology allows for a comprehensive although brief picture of contemporaneous usual care practice. Simultaneous inclusion of a wide range of acute facilities, ranging from tertiary urban referral centres to small rural and district hospitals, helps to ensure a real world representation of patients with acute decompensated HF.

Although this was the first population-based point prevalence survey of acute HF admissions in Australia, several limitations must be acknowledged. Hospitals were invited to participate, and all sites were teaching hospitals with staff cardiologists, and may therefore not be representative of remote facilities or hospitals without an on-site cardiologist. As the case report form consisted of only two pages, it was not possible to collect detailed information in all fields, such as echocardiography results and the aetiology of HF. As we were unable to collect data from non-participating institutions, the length of stay for patients transferred from or to another facility may have been underestimated. Finally, the NSW HF Snapshot was conducted during a one-month period during the Australian winter; as some risk factors may vary seasonally, our survey may not accurately reflect patterns of admission and care throughout the year.

\section{Conclusion}

The NSW HF Snapshot has highlighted the advanced age and multiple comorbidities of patients admitted to NSW and ACT hospitals with acute $\mathrm{HF}$, and the high prevalence of frailty in these patients. The use of evidence-based therapies for patients with decompensated $\mathrm{HF}$, particularly ACEIs and $\beta$-blockers, was less than optimal, and there was little evidence that these therapies were introduced during the patients' stays in hospital. The extent to which patient, institutional and system characteristics contribute to variations in care and outcomes is being explored in further analyses. We anticipate that the results of the NSW HF Snapshot will inform the development of strategies for improving access to multidisciplinary care programs and increasing the uptake of evidence-based therapies.

Acknowledgements: The NSW HF Snapshot investigators gratefully acknowledge all participating patients, the site coordinators who collected the data, and Angela Brennan, Philippa Loane and staff at Monash University, who undertook data management. The NSW HF Snapshot study was funded by the National Heart Foundation New South Wales Cardiovascular Research Network. Emily Banks is supported by the National Health and Medical Research Council of Australia.

Competing interests: No relevant disclosures.

Received 13 Jul 2015, accepted 23 Nov 2015. 口

(c) 2016 AMPCo Pty Ltd. Produced with Elsevier B.V. All rights reserved. 
1 Horowitz JD, Stewart S. Heart failure in older people: the epidemic we had to have. Med J Aust 2001; 174: 432-433. https://www.mja.com.au/journal/ 2001/174/9/heart-failure-older-peopleepidemic-we-had-have

2 Phillips SM, Marton RL, Tofler GH. Barriers to diagnosing and managing heart failure in primary care. Med $J$ Aust 2004; 181: 78-81. https://www.mja.com. au/journal/2004/181/2/barriersdiagnosing-and-managing-heartfailure-primary-care

3 Chew DP, French J, Briffa TG, et al. Acute coronary syndrome care across Australia and New Zealand: the SNAPSHOT ACS study. Med J Aust 2013; 199: 185-191. https://www.mja. com.au/journal/2013/199/3/acutecoronary-syndrome-care-acrossaustralia-and-new-zealand-snapshotacs-study

4 McKee PA, Castelli WP, McNamara PM, et al. The natural history of congestive heart failure: the Framingham study. N Engl J Med 1971; 285: 1441-1446.

5 Quan $\mathrm{H}$, Li B, Couris CM, et al. Updating and validating the Charlson comorbidity index and score for risk adjustment in hospital discharge abstracts using data from 6 countries. Am J Epidemiol 2011; 173: 676-682.

6 Romero-Ortuno R, Walsh CD, Lawlor BA, et al. A frailty instrument for primary care: findings from the Survey of Health, Ageing and Retirement in Europe (SHARE). BMC Geriatr 2010; 10: 57.

7 Follath F, Yilmaz M, Delgado J, et al. Clinical presentation, management and outcomes in the acute heart failure global survey of standard treatment (ALARM-HF). Intensive Care Med 2011; 37: 619-626.

8 Sato N, Kajimoto K, Keida T, et al. Clinical features and outcome in hospitalized heart failure in Japan (from the ATTEND Registry). Circ $J$ 2013; 77: 944-951.

9 Spinar J, Parenica J, Vitovec J, et al. Baseline characteristics and hospital mortality in the Acute Heart Failure Database (AHEAD) Main registry. Crit Care 2011; 15: R291.

10 Chioncel O, Vinereanu D, Datcu M, et al. The Romanian Acute Heart Failure
Syndromes (RO-AHFS) registry. Am Heart J 2011; 162: 142-153.el.

11 Zannad F, Mebazaa A, Juillière $Y$, et al. Clinical profile, contemporary management and one-year mortality in patients with severe acute heart failure syndromes: the EFICA study. Eur J Heart Fail 2006; 8: 697-705.

12 Oliva F, Mortara A, Cacciatore G, et al. Acute heart failure patient profiles, management and in-hospital outcome: results of the Italian Registry on Heart Failure Outcome. Eur J Heart Fail 2012; 14: 1208-1217.

13 Maggioni AP, Dahlström U, Filippatos G, et al. EURObservational Research Programme: The Heart Failure Pilot Survey (ESC-HF Pilot). Eur J Heart Fail 2010; 12: 1076-1084.

14 Nieminen MS, Brutsaert D, Dickstein K, et al. EuroHeart Failure Survey II (EHFS II): a survey on hospitalized acute heart failure patients: description of population. Eur Heart J 2006; 27: 2725-2736.

15 Steinberg BA, Zhao X, Heidenreich PA, et al. Trends in patients hospitalized with heart failure and preserved left ventricular ejection fraction: prevalence, therapies, and outcomes. Circulation 2012; 126: 65-75.

16 Gheorghiade M, Abraham WT, Albert NM, et al. Systolic blood pressure at admission, clinical characteristics, and outcomes in patients hospitalized with acute heart failure. JAMA 2006; 296: 2217-2226.

17 Adams KF, Fonarow GC, Emerman CL, et al. Characteristics and outcomes of patients hospitalized for heart failure in the United States: rationale, design, and preliminary observations from the first 100,000 cases in the Acute Decompensated Heart Failure National Registry (ADHERE). Am Heart J 2005; 149: 209-216.

18 Atherton JJ, Hayward CS, Ahmad WAW, et al. Patient characteristics from a regional multicenter database of acute decompensated heart failure in Asia Pacific (ADHERE International-Asia Pacific). J Card Fail 2012; 18: 82-88.

19 Krum H, Tonkin AM, Currie R, et al. Chronic heart failure in Australian general practice. The Cardiac Awareness Survey and Evaluation
(CASE) Study. Med J Aust 2001; 174: 439-444. https://www.mja.com.au/ journal/2001/174/9/chronic-heartfailure-australian-general-practice

20 Tsuyuki RT, McKelvie RS, Arnold JM, et al. Acute precipitants of congestive heart failure exacerbations. Arch Intern Med 2001; 161: 2337-2342.

21 Page K, Marwick TH, Lee $R$, et al. A systematic approach to chronic heart failure care: a consensus statement. Med J Aust 2014; 201: 146-150. https:// www.mja.com.au/journal/2014/201/3/ systematic-approach-chronic-heartfailure-care-consensus-statement

22 Ambrosy AP, Fonarow GC, Butler J, et al. The global health and economic burden of hospitalizations for heart failure. Lessons learned from hospitalized heart failure registries. J Am Coll Cardiol 2014; 63: 1123-1133.

23 Fonarow GC, Heywood JT, Heidenreich PA, et al. Temporal trends in clinical characteristics, treatments, and outcomes for heart failure hospitalizations, 2002 to 2004: findings from Acute Decompensated Heart Failure National Registry (ADHERE). Am Heart J 2007; 153: 1021-1028.

24 Costanzo MR, Johannes RS, Pine M, et al. The safety of intravenous diuretics alone versus diuretics plus parenteral vasoactive therapies in hospitalized patients with acutely decompensated heart failure: a propensity score and instrumental variable analysis using the Acutely Decompensated Heart Failure National Registry (ADHERE) database. Am Heart J 2007; 154: 267-277.

25 Krum H, Jelinek M, Stewart S, et al; on behalf of the CHF Guidelines Core Writers. Guidelines for the prevention, detection and management of people with chronic heart failure in Australia 2006. Med J Aust 2006; 185: 549-556. https://www.mja.com.au/ journal/2006/185/10/guidelinesprevention-detection-andmanagement-people-chronic-heartfailure

26 Jha S, Ha S, Hickman L, et al. Frailty in advanced heart failure: a systematic review. Heart Fail Rev 2015; 20: 553-560. 